\section{- OPEN ACCESS}

\title{
Genetic mannose binding lectin deficiency is associated with airway microbiota diversity and reduced exacerbation frequency in COPD
}

\author{
Alison J Dicker, ${ }^{1}$ Megan L Crichton, ${ }^{1}$ Andrew J Cassidy, ${ }^{1}$ Gill Brady, ${ }^{2}$ Adrian Hapca, ${ }^{3}$ \\ Roger Tavendale, ${ }^{4}$ Gisli G Einarsson, ${ }^{5}$ Elizabeth Furrie, ${ }_{1}^{6}$ J Stuart Elborn, ${ }^{5,7}$ \\ Stuart Schembri, ${ }^{1}$ Sara E Marshall, ${ }^{1}$ Colin N A Palmer, ${ }^{4}$ James D Chalmers ${ }^{1}$
}

\begin{abstract}
- Additional material is published online only. To view please visit the journal online (http://dx.doi.org/10.1136/ thoraxjnl-2016-209931).

For numbered affiliations see end of article.
\end{abstract}

\section{Correspondence to} Dr James D Chalmers, Scottish Centre for Respiratory Research, University of Dundee, Ninewells Hospital \& Medical School, Dundee DD1 9SY, UK jchalmers@dundee.ac.uk

Received 26 December 2016 Revised 19 August 2017 Accepted 2 October 2017 Published Online First 3 November 2017

\section{Linked}

http://dx.doi.org/10.1136/ thoraxjnl-2017-211106

Check for updates

To cite: Dicker AJ,

Crichton ML, Cassidy AJ, et al. Thorax 2018:73:510-518.

\section{ABSTRACT}

Background In cystic fibrosis and bronchiectasis, genetic mannose binding lectin (MBL) deficiency is associated with increased exacerbations and earlier mortality; associations in COPD are less clear. Preclinical data suggest MBL interferes with phagocytosis of Haemophilus influenzae, a key COPD pathogen. We investigated whether MBL deficiency impacted on clinical outcomes or microbiota composition in COPD.

Methods Patients with COPD ( $n=1796)$ underwent MBL genotyping; linkage to health records identified exacerbations, lung function decline and mortality. A nested subcohort of 141 patients, followed for up to 6 months, was studied to test if MBL deficiency was associated with altered sputum microbiota, through 165 rRNA PCR and sequencing, or airway inflammation during stable and exacerbated COPD.

Findings Patients with MBL deficiency with COPD were significantly less likely to have severe exacerbations (incidence rate ratio (IRR) $0.66,95 \% \mathrm{Cl} 0.48$ to 0.90 , $p=0.009$ ), or to have moderate or severe exacerbations (IRR $0.77,95 \% \mathrm{Cl} 0.60$ to $0.99, p=0.047$ ). MBL deficiency did not affect rate of FEV decline or mortality. In the subcohort, patients with MBL deficiency had a more diverse lung microbiota $(p=0.008)$, and were less likely to be colonised with Haemophilus spp. There were lower levels of airway inflammation in patients with MBL deficiency.

Interpretation Patients with MBL deficient genotype with COPD have a lower risk of exacerbations and a more diverse lung microbiota. This is the first study to identify a genetic association with the lung microbiota in COPD.

\section{INTRODUCTION}

Chronic obstructive pulmonary disease (COPD) is characterised by airway inflammation, with periods of symptom exacerbation, leading to irreversible airflow limitation, lung damage and mucus hypersecretion. COPD has multiple phenotypes and an unpredictable clinical course. ${ }^{1}$ Culture independent techniques have shown that lungs of healthy adults and patients with COPD harbour a diverse microbiota. ${ }^{2}$ It is likely that genetic influences affect disease outcomes and microbiota composition through modulation of host defence mechanisms. There are few studies of genetic modifiers of

\section{Key messages}

What is the key question?

- COPD is a heterogeneous disease with airway inflammation being driven by bacterial infections, yet no studies to date have examined microbiology or infection risk in COPD in relation to mannose binding lectin (MBL) genotype.

What is the bottom line?

- We identified that genetic MBL deficiency was protective against exacerbation risk in a large population of patients with COPD. We explored the mechanism of this effect and identified that MBL deficiency was associated with reduced presence of Haemophilus spp in the airway microbiota.

Why read on?

- This is one of the largest studies of the COPD lung microbiota to date, and is the first study to demonstrate that MBL deficiency is a key contributor to infection risk in COPD.

exacerbation risk in COPD, and no studies relating host genotype with lung microbiota. ${ }^{34}$

Mannose binding lectin (MBL), an innate immune system component, activates the lectin complement pathway and opsonises organisms for phagocytosis; it accumulates in the lungs during inflammation. ${ }^{5}$ Individuals with specific single nucleotide polymorphisms (SNPs) of the MBL2 gene cannot form stable multimeric forms, leading to systemic deficiency of the MBL protein. ${ }^{6}$ Of the population, $10 \%-30 \%$ carry MBL deficient haplotypes but have no detectable phenotype when healthy. ${ }^{7}$ In populations with bronchiectasis or cystic fibrosis (CF), MBL deficiency is associated with more exacerbations (including severe exacerbations), faster decline in $\mathrm{FEV}_{1}$ and increased Pseudomonas aeruginosa colonisation. ${ }^{78}$ In COPD, associations between MBL deficiency and hospitalisations for acute exacerbations or more frequent exacerbations have been identified, ${ }^{9} 10$ whereas Eagan $e t a l^{11}$ found no relationship between MBL plasma levels and COPD risk or disease severity. It could be assumed that MBL deficiency in COPD, 
$\mathrm{CF}$ or bronchiectasis would have similar effects but there are clear differences in the inflammatory profiles and microbiota of these diseases. MBL undergoes oxidation in the COPD airway; oxidised MBL suppresses macrophage phagocytosis of Haemophilus influenzae, a key COPD pathogen. ${ }^{12}$ Failed macrophage phagocytosis in COPD has been extensively described and is likely to contribute to exacerbation risk. ${ }^{13}$ Paradoxically, MBL deficiency could be beneficial in COPD, by removing a key inhibitor of macrophage function and bacterial clearance. This study was designed to test if MBL deficiency protects against exacerbations and is associated with a lower abundance of Haemophilus spp in the COPD airway.

\section{METHODS}

\section{Tayside Allergy and Respiratory Disease Information System} population based genetic study

In 2008, patients age $>40$ years; with an $\mathrm{FEV}_{1} / \mathrm{FVC}$ ratio $<70 \%$ and a clinical diagnosis of COPD were invited to participate in a primary care based longitudinal cohort study and population registry called Tayside Allergy and Respiratory Disease Information System (TARDIS). Patients were reviewed annually for lung function performance, recording of symptoms and exacerbation history. The inclusion criteria were age $>40$ years, a postbronchodilator $\mathrm{FEV}_{1} / \mathrm{FVC}$ ratio of $<70 \%$ and a clinical diagnosis of COPD. Record linkage was used to obtain prescribing history, hospitalisations, morbidity and death, using the Scottish morbidity database, the Tayside community prescription database and the General Register Office for Scotland. Patients gave informed consent to participate. Saliva was collected for DNA extraction using Oragene kits (DNA Genotek). In the present analysis we excluded patients with a primary diagnosis of bronchiectasis, asthma or immunodeficiency.

\section{SNP genotyping}

TARDIS DNA samples were genotyped for four MBL2 SNPs (promoter region SNP rs7096206, and exon 1 SNPs rs1800450, rs1800451, rs5030737) which previous studies have used to define genetic MBL deficiency. ${ }^{6}$ Further details of SNP genotyping are shown in the online supplementary material . Patients were classified as deficient, intermediate or sufficient MBL genotype according to generally accepted definitions. $^{6}$

\section{TARDIS outcomes}

The primary outcome was to determine if MBL deficiency was associated with frequency of exacerbations. Exacerbations were recorded annually and validated against prescribing and hospital admission data. Local guidelines for COPD exacerbation management recommend $30-40 \mathrm{mg} /$ day prednisolone with antibiotics prescribed in the presence of purulent sputum. Exacerbations were identified as an acute prescription for $>20 \mathrm{mg} /$ day of prednisolone (moderate exacerbations) and/or admission to hospital due to exacerbation of COPD (severe exacerbation). Prescribing data were used to distinguish between those exacerbations treated with corticosteroids alone or those also treated with antibiotics. Statistical models were constructed comparing MBL sufficient, intermediate and deficient genotypes (three level model) and comparing MBL deficient to non-deficient (intermediate and sufficient combined) genotypes (two level model). Secondary outcomes investigated were decline in $\mathrm{FEV}_{1}$ over time, admissions to hospital for cardiovascular events (including myocardial infarction, cardiac failure and arrhythmias) and all-cause, respiratory or cardiovascular mortalities.

\section{Microbiota subcohort study}

Subsequently, a nested cohort of patients was recruited, based on MBL genotype, into a more detailed study to identify the impact of MBL deficiency on exacerbation risk. Patients with known MBL genotype, who were alive in 2013 and had given consent to be contacted again for research studies were approached by letter. Those that responded to the invitation were screened and included in the subcohort study.

Patients enrolled during a period of disease stability were followed up for between 3 months to 6 months. During this period, patients contacted the study team in the event of an exacerbation as defined in the online supplementary material. ${ }^{14}$ Blood and sputum samples for analysis of systemic and airway inflammation, and for examination of lung microbiota, were taken at the beginning and end of the study (when clinically stable) and at the beginning and 10 days after the start of exacerbations. Inclusion and exclusion criteria and study procedures are shown in the online supplementary material.

\section{Systemic and airway inflammation}

Sputum and serum biomarkers (interleukin (IL) $1 \beta$, IL-8 and tumour necrosis factor $\alpha(\mathrm{TNF} \alpha)$, extracellular newly identified receptor for advanced glycation end products binding protein (EN-RAGE), myeloperoxidase, C-reactive protein (CRP), CD40L, P-selectin) were measured by ELISA as described in the online supplementary material.

\section{Sputum microbiota}

DNA extracted from whole sputum underwent PCR and sequencing of the bacterial 16S rRNA gene on the Illumina MiSeq followed by bioinformatic analysis in the Quantitative Insights Into Microbial Ecology pipeline V.1.9.0 as described in the online supplementary material.

\section{Bacterial binding studies}

The ability of MBL and the closely related molecules ficolins 2 and 3 to bind to clinically relevant respiratory pathogens were assessed in vitro as detailed in the online supplementary material.

\section{Statistical analysis}

Descriptive data are expressed as mean $\pm \mathrm{SD}$ or count with percentage as appropriate. Sample size was empirical based on the number of available patients within the TARDIS cohort. Exacerbation frequency was evaluated using negative binomial models accounting for differing lengths of follow-up per patient. Survival was assessed by Cox proportional hazards regression from date of enrolment to death. Comparison of the average annual rate in decline of $\mathrm{FEV}_{1}$ between MBL deficient, intermediate and sufficient patients was performed using multiple linear regression. The annualised rate of $\mathrm{FEV}_{1}$ decline was determined from the difference between the $\mathrm{FEV}_{1}$ recorded at the final TARDIS visit and the baseline visit divided by follow-up time. Multivariable models were adjusted for gender, Deprivation Index, prescriptions for inhaled corticosteroids (ICS), bronchodilators and other medications (listed in table 1), smoking status and pack years, $\mathrm{FEV}_{1}$, post bronchodilator $\mathrm{FEV}_{1}$, comorbidities of cardiovascular disease, liver disease, renal disease, hypertension and diabetes. Data were obtained from record linkage and so no missing data were encountered for prescribing, morbidity and mortality data. Subgroups were predefined including examining 
Table 1 Table showing the baseline characteristics of the Tayside Allergy and Respiratory Disease Information System (TARDIS) cohort analysis by mannose binding lectin (MBL) genotype. $p$ Values were calculated by three-way comparisons (Kruskal-Wallis for nonparametrical data, analysis of variance for parametrical data and $\mathrm{X}^{2}$ test for categorical data) across all groups

\begin{tabular}{|c|c|c|c|c|}
\hline & $\begin{array}{l}\text { MBL deficient } \\
\text { genotype }\end{array}$ & $\begin{array}{l}\text { MBL } \\
\text { intermediate } \\
\text { genotype }\end{array}$ & $\begin{array}{l}\text { MBL } \\
\text { sufficient } \\
\text { genotype }\end{array}$ & $\mathrm{p}$ Value \\
\hline $\mathrm{N}$ (\% of study cohort) & $240(13.4)$ & $586(32.6)$ & $970(54.0)$ & $\mathrm{n} / \mathrm{a}$ \\
\hline $\begin{array}{l}\text { Average follow-up } \\
\text { length in years (SD) }\end{array}$ & $5.44( \pm 2.1)$ & $5.34( \pm 2.0)$ & $5.43( \pm 2.1)$ & 0.9 \\
\hline $\begin{array}{l}\text { Age at } \\
\text { diagnosis, years (SD) }\end{array}$ & $65.6( \pm 9.0)$ & $64.23( \pm 9.6)$ & $64.4( \pm 9.6)$ & 0.1 \\
\hline $\begin{array}{l}\text { Male gender (\% of } \\
\text { group) }\end{array}$ & $131(54.6)$ & $308(52.7)$ & $506(52.2)$ & 0.8 \\
\hline $\begin{array}{l}\text { Cigarette smoking by } \\
\text { pack years (SD) }\end{array}$ & $41.6( \pm 20.6)$ & $41.0( \pm 20.3)$ & $41.3( \pm 22.7)$ & 0.7 \\
\hline Body mass index (SD) & $27.0( \pm 5.6)$ & $26.9( \pm 5.4)$ & $27.2( \pm 5.6)$ & 0.9 \\
\hline $\mathrm{FEV}_{1} \%$ predicted (SD) & $79.6( \pm 20.8)$ & $78.7( \pm 24.4)$ & $78.0( \pm 22.1)$ & 0.3 \\
\hline $\begin{array}{l}\text { MRC Dyspnoea Score } \\
\text { (SD) }\end{array}$ & $2.44( \pm 1.0)$ & $2.53( \pm 1.0)$ & $2.48( \pm 1.0)$ & 0.6 \\
\hline $\mathrm{FEV}_{1} / \mathrm{FVC}$ (SD) & $0.59( \pm 0.1)$ & $0.58( \pm 0.1)$ & $0.58( \pm 0.1)$ & 0.3 \\
\hline \multicolumn{5}{|l|}{ Comorbidities } \\
\hline $\begin{array}{l}\text { Cardiovascular } \\
\text { disease (\% of group) }\end{array}$ & $24(10.0)$ & $53(9.0)$ & $78(8.0)$ & 0.6 \\
\hline $\begin{array}{l}\text { Renal failure (\% of } \\
\text { group) }\end{array}$ & $5(2.1)$ & $17(2.9)$ & $19(2.0)$ & 0.5 \\
\hline Cancer (\% of group) & $12(5.0)$ & $29(4.9)$ & $39(4.0)$ & 0.6 \\
\hline $\begin{array}{l}\text { Hypertension (\% of } \\
\text { group) }\end{array}$ & $137(57.1)$ & $322(54.9)$ & $524(54.0)$ & 0.7 \\
\hline Diabetes (\% of group) & $48(20.0)$ & $105(17.9)$ & $194(20.0)$ & 0.6 \\
\hline \multicolumn{5}{|l|}{ Therapies } \\
\hline $\begin{array}{l}\text { Inhaled corticosteroids } \\
\text { (\% of group) }\end{array}$ & $123(51.3)$ & 349 (59.6) & $570(58.8)$ & 0.1 \\
\hline $\begin{array}{l}\text { Long-acting } \\
\text { muscarinic antagonist } \\
\text { (\% of group) }\end{array}$ & $67(27.9)$ & $175(29.9)$ & $257(26.5)$ & 0.4 \\
\hline Statins ( $\%$ of group) & $71(29.6)$ & $178(30.4)$ & $313(32.3)$ & 0.6 \\
\hline
\end{tabular}

exacerbations requiring corticosteroids versus those requiring antibiotics, and moderate versus severe exacerbations (defined as those requiring hospital admission). All models used time-dependent analysis to account for changes in these variables over the course of follow-up. Differences between effect estimates were compared using interaction testing. ${ }^{15}$ Analysis of stable samples used only the first stable sample per patient. All statistical analyses were carried out using SAS V.9.3 or Graphpad Prism V.5.

\section{RESULTS \\ TARDIS population-based genetic study}

A flow chart detailing the numbers of samples in both cohorts is shown in figure 1, with 1796 patients included in this analysis; $53 \%$ were male, an average age of 64.5 years, a mean $\mathrm{FEV}_{1}$ of $78 \%$ predicted, an $\mathrm{FEV}_{1} / \mathrm{FVC}$ ratio of 0.58 and a smoking history of 41.26 pack years. Table 1 gives details of the cohort at baseline, split according to MBL genotype. There were no statistically significant differences in age, gender, $\mathrm{FEV}_{1}$, body mass

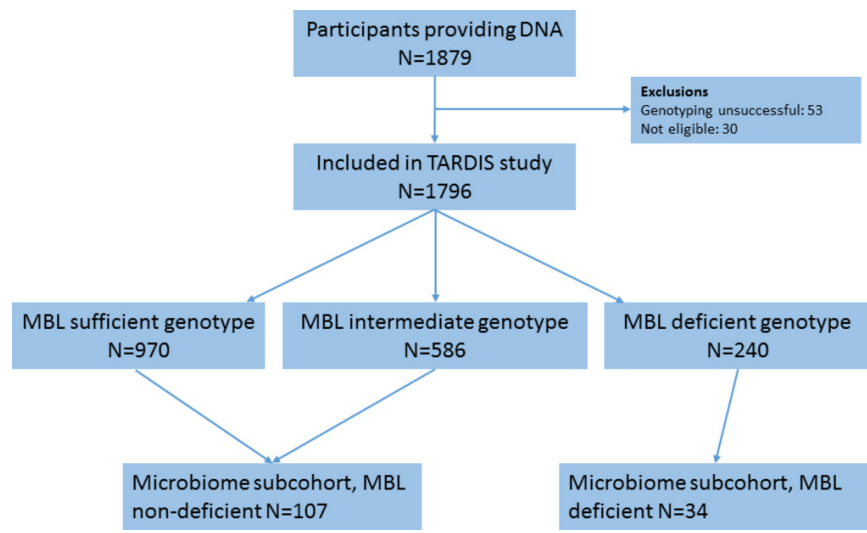

Figure 1 Flow chart of samples through the Tayside Allergy and Respiratory Disease Information System (TARDIS) and microbiome subcohort studies. MBL, mannose binding lectin.

index, comorbidities and smoking status between the groups. Mean follow-up time was 5.4 years.

\section{MBL deficiency is associated with reduced exacerbation frequency but not mortality in COPD}

During follow-up the rate of exacerbations per patient per year was 2.52/year in the MBL sufficient haplotype group and 1.70/ year in patients with MBL deficient haplotypes. In the adjusted negative binomial model, MBL deficient haplotypes were associated with 23\% fewer exacerbations (figure 2) compared with MBL sufficient individuals. A significant reduction in exacerbation frequency was observed when comparing MBL deficient versus sufficient genotypes, or when comparing patients with MBL deficiency with all other groups (incidence rate ratio (IRR) $0.74,95 \%$ CI 0.56 to $0.98, \mathrm{p}=0.04$ ). The effect was greatest for severe exacerbations; MBL deficiency reduced the likelihood of hospitalisation by $34 \%$ (IRR $0.66,95 \%$ CI 0.48 to 0.90 , $\mathrm{p}=0.009$ ), although there was no statistically significant difference on interaction testing $(p=0.7)$, indicating no differential effect of MBL deficiency on severe and non-severe exacerbations. Sensitivity analysis showed the reduction in exacerbations was driven by a reduction in infective exacerbations requiring antibiotics (figure 2). The rates of $\mathrm{FEV}_{1}$ decline were $38.4 \mathrm{~mL} /$ year in patients with MBL deficient haplotypes, $45.1 \mathrm{~mL} /$ year in those with intermediate haplotypes and $45.0 \mathrm{~mL} /$ year in those with sufficient haplotypes. In the adjusted linear regression model MBL deficiency was not associated with a more rapid decline in $\mathrm{FEV}_{1}$ over time (estimate 0.27 , SE $0.31, \mathrm{p}=0.4$ ). Similar results were obtained from the three-level and two-level models.

There were 589 deaths from any cause during follow-up (32.8\% of the cohort). Three hundred and sixty deaths were classified as respiratory related, 133 were classified as cardiovascular and 96 were from other causes. The corresponding death rates for MBL deficient versus non-deficient groups were 31.3\% versus $33.1 \%$ for all-cause mortality, $17.5 \%$ versus $20.5 \%$ for respiratory mortality and $6.3 \%$ versus $7.6 \%$ for cardiovascular deaths. In the adjusted analysis, MBL deficiency (deficient vs non-deficient) was not associated with respiratory (HR 0.83 , $95 \%$ CI 0.56 to $1.35, \mathrm{p}=0.4$ ), cardiovascular (HR $0.81,95 \% \mathrm{CI}$ 0.44 to $1.48, \mathrm{p}=0.5$ ) or all-cause mortalities (HR $0.94,95 \% \mathrm{CI}$ 0.72 to $1.24, p=0.7$ ) (figure 2 ). Similar results were obtained using the three-level model. Intermediate expressing haplotypes were not associated with increased exacerbation frequency compared with sufficient haplotypes (IRR $0.98,95 \%$ CI 0.82 to $1.18, \mathrm{p}=0.8)$. 


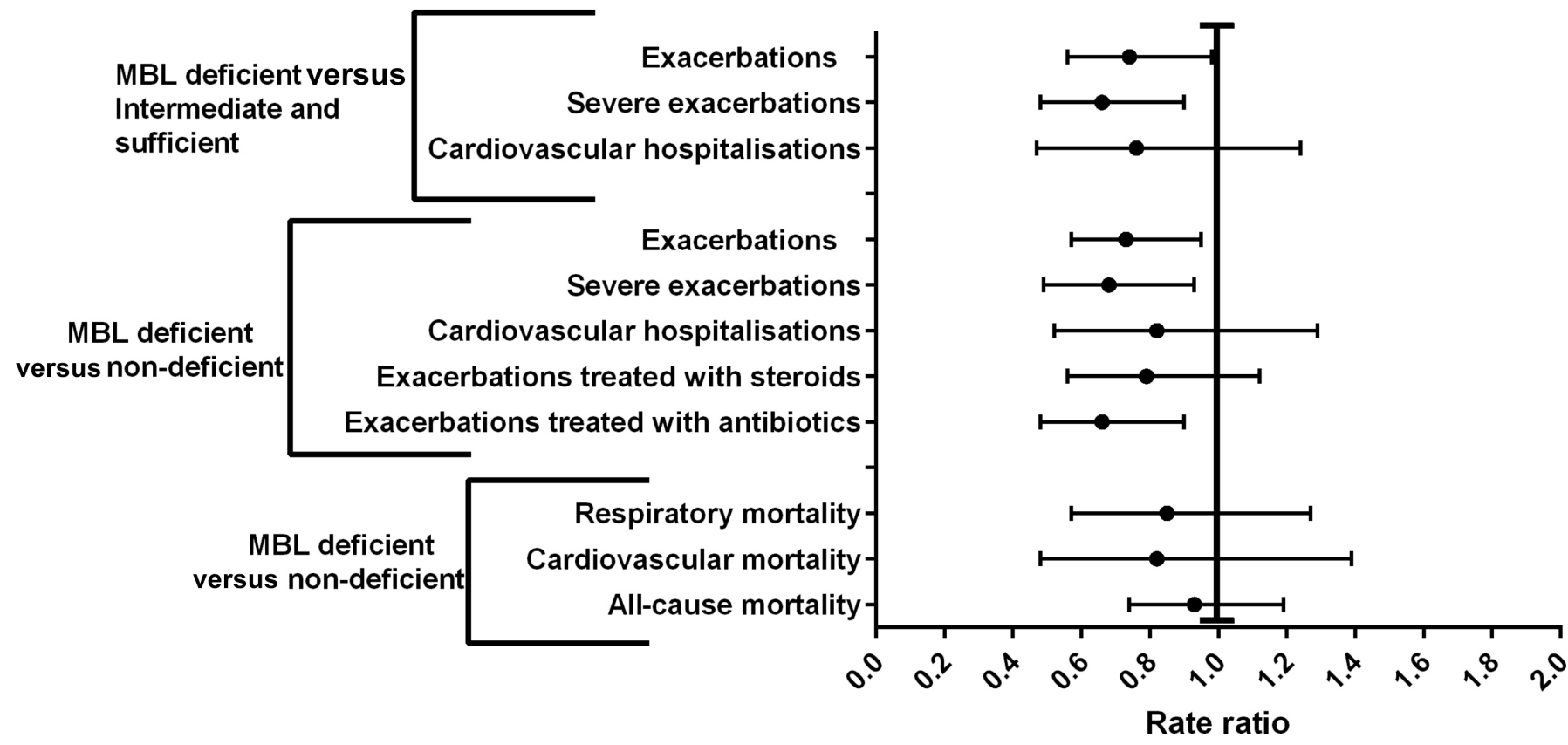

Figure 2 Risk of exacerbations, cardiovascular hospitalisations and mortality according to mannose binding lectin (MBL)2 genotype. Two models were made comparing MBL sufficient, intermediate and deficient genotypes (three-level model) and comparing MBL deficient to non-deficient (intermediate and sufficient combined) genotypes (two-level model). A rate ratio $<1$ indicates a lower risk of exacerbation, hospitalisation or mortality with low expressing genotypes. Results are significant at $p<0.05$ where the $95 \% \mathrm{Cl}$ does not cross a ratio of 1.0 .

\section{Microbiota subcohort study}

Based on the finding of less frequent and fewer infective exacerbations in patients with MBL deficiency, we recruited two subcohorts of patients with TARDIS COPD by MBL genotype to compare the microbiota and inflammatory profiles between these subcohorts (figure 1, $n=141$ ). Thirty-four patients were classified as MBL deficient and 107 as MBL non-deficient by genotypes. At baseline, patients with MBL deficient and non-deficient genotypes were well matched in terms of Medical Research Council (MRC) Dyspnoea Score, quality of life (St George's Respiratory Questionnaire), symptoms (COPD assessment test), Global Obstructive Lung Disease (GOLD) 2011 class and per cent predicted $\mathrm{FEV}_{1}$ (table 2).

\section{MBL deficiency is associated with an increased diversity in microbiota}

Forty-two patients could not provide sputum samples during the study; comparisons between sputum producers and the whole cohort (online supplementary table E1) indicated there was no significant difference in baseline characteristics between sputum producers and non-producers. Two hundred and ten sputum samples were obtained; 184 sputum samples were of sufficient quality and quantity for successful bacterial DNA extraction and 16S rRNA gene sequencing. One hundred and twenty-six samples passed the subsequent data analysis quality control cut-offs; 89 were from patients when clinically stable, 16 were start of exacerbation and 21 were end of exacerbation samples.

Figure 3A shows one representative stable microbiota from each patient, alongside a comparison of the averaged microbiota profiles for the MBL deficient versus non-deficient populations (figure $3 \mathrm{~B}$ ). $\alpha$ Diversity (a measure of the evenness and richness of the microbiota of individual samples) was determined by Shannon-Wiener Diversity Index (S-WDI); patients with MBL deficiency had a more diverse lung microbiota than patients without MBL deficiency $(p=0.008$, figure 3D) and were less likely to be colonised with Haemophilus spp (figure 4B, $\mathrm{p}=0.043$ ). Decreasing $\alpha$ diversity (lower S-WDI) was associated with more severe GOLD Score (figure 3E, $\mathrm{p}=0.045$ ), with two clusters of S-WDI being driven by the percentage of operational taxonomic units (OTUs) identified as Haemophilus spp (figure $3 \mathrm{~F}$ ). The $\beta$ diversity, a measurement of how different individual samples microbiota are from each other, of all stable samples is shown in the online supplementary figure E1.

\section{Haemophilus spp dominance is associated with disease} severity and increased airway inflammation

The dominant Haemophilus spp in $39.2 \%$ of the stable patients, identified by a BLASTn search (https://blast.ncbi.nlm.nih.gov/ Blast.cgi) of representative sequences of each Haemophilus OTU, was H. influenzae (figure 4A). A cut-off of 40\% Haemophilus spp OTUs (based on figure $3 \mathrm{~F}$ ) was used to stratify patients as Haemophilus spp dominant and compared with markers of disease severity and exacerbation frequency (figure 4C,D). A more severe GOLD Score and more exacerbations per year were associated with having $>40 \%$ Haemophilus spp OTUs (both $\mathrm{p}=0.03)$.

Lung inflammatory profiles, including IL-1 $\beta$ (previously shown to distinguish between infectious and non-infectious drivers of inflammation in $\mathrm{COPD}^{16}$ ), were compared between samples with less than or greater than $40 \%$ Haemophilus spp OTUs. Significantly higher levels of IL-1 $\beta(p<0.0001)$ and TNF $\alpha(p<0.0001)$ were observed in sputum samples from stable patients with $>40 \%$ Haemophilus spp OTUs (figure 4E,F). Systemic markers of inflammation or sputum myeloperoxidase, EN-RAGE or IL-8 were not significantly different in patients categorised according to \% Haemophilus spp OTUs. 
Table 2 Baseline characteristics of the patients with mannose binding lectin (MBL) deficiency and patients without $\mathrm{MBL}$ deficiency in the microbiome subcohort study. GOLD class was calculated based on the 2011 guidelines. $p$ Values were calculated through pairwise comparisons by $T$ test for parametrical data and Mann-Whitney $U$ test for non-parametrical data

\begin{tabular}{|c|c|c|c|}
\hline & $\begin{array}{l}\text { MBL deficient } \\
\text { genotypes }\end{array}$ & $\begin{array}{l}\text { MBL non-deficient } \\
\text { genotypes }\end{array}$ & $\mathrm{p}$ Value \\
\hline \multicolumn{4}{|c|}{ Demographics and major comorbidities } \\
\hline $\mathrm{N}$ & 34 & 107 & \\
\hline Age, years (SD) & $72.8( \pm 7.3)$ & $70.8 \pm(8.2)$ & 0.2 \\
\hline Age at diagnosis, years (SD) & $59.5( \pm 9.1)$ & $60.2( \pm 11.9)$ & 0.8 \\
\hline Male gender (\%) & $22(64.7)$ & $67(62.6)$ & 0.8 \\
\hline Active smokers (\%) & $7(20.6)$ & $21(19.6)$ & 0.9 \\
\hline $\begin{array}{l}\text { Cigarette smoking by pack } \\
\text { years (SD) }\end{array}$ & $45.1( \pm 29.9)$ & $41.7( \pm 28.9)$ & 0.6 \\
\hline Body mass index (SD) & $28.2( \pm 5.3)$ & $28.1( \pm 5.6)$ & 0.9 \\
\hline Myocardial Infarction (\%) & $4(12.1)$ & $12(11.2)$ & 0.9 \\
\hline CABG (\%) & $3(8.8)$ & $12(11.2)$ & 0.7 \\
\hline Angina (\%) & $6(18.2)$ & $24(22.4)$ & 0.6 \\
\hline Stroke $(\%)$ & $7(20.6)$ & $6(5.6)$ & 0.008 \\
\hline Diabetes (\%) & $5(14.7)$ & $22(20.6)$ & 0.4 \\
\hline \multicolumn{4}{|l|}{ COPD characteristics } \\
\hline FEV ${ }_{1}$ per cent predicted (SD) & $65.0( \pm 19.4)$ & $72.1( \pm 20.7)$ & 0.08 \\
\hline MRC Dyspnoea Score (SD) & $3.0( \pm 1.5)$ & $2.58( \pm 1.4)$ & 0.1 \\
\hline Exacerbations per year (SD) & $1.5( \pm 1.5)$ & $1.9( \pm 1.9)$ & 0.3 \\
\hline Blood eosinophils > 2\% (\%) & $16(47.1)$ & $70(65.4)$ & 0.06 \\
\hline GOLD Score & & & $0.6^{*}$ \\
\hline A (\%) & $2(5.9)$ & $15(14.0)$ & - \\
\hline B (\%) & $14(41.2)$ & $38(35.5)$ & - \\
\hline C (\%) & $2(5.9)$ & $4(3.7)$ & - \\
\hline $\mathrm{D}(\%)$ & $16(47.1)$ & $50(46.7)$ & - \\
\hline SGRQ (SD) & $47.8( \pm 23.3)$ & $39.7( \pm 22.6)$ & 0.07 \\
\hline On LTOT (\%) & $0(0)$ & $5(4.7)$ & 0.2 \\
\hline \multicolumn{4}{|l|}{ Medications } \\
\hline ICS/LABA (\%) & $22(64.7)$ & $62(57.9)$ & 0.5 \\
\hline LABA alone (\%) & $3(8.8)$ & $13(12.1)$ & 0.6 \\
\hline LAMA (\%) & $19(55.9)$ & $51(47.7)$ & 0.4 \\
\hline Mucolytic (\%) & $7(20.6)$ & $10(9.3)$ & 0.08 \\
\hline Aspirin (\%) & $10(29.4)$ & $27(25.2)$ & 0.6 \\
\hline$\beta$ blocker (\%) & $5(14.7)$ & $13(12.1)$ & 0.7 \\
\hline Statin (\%) & $20(58.8)$ & $56(52.3)$ & 0.5 \\
\hline ACE inhibitor (\%) & $7(20.6)$ & $30(28.0)$ & 0.4 \\
\hline Clopidogrel (\%) & $3(8.8)$ & $7(6.5)$ & 0.7 \\
\hline
\end{tabular}

*indicates $\mathrm{X}^{2}$ test across all four GOLD groups.

CABG, coronary artery bypass graft; GOLD, Global Obstructive Lung Disease; ICS, inhaled corticosteroids; LABA, long-acting $\beta$ agonist; LAMA, long-acting muscarinic antagonist; LTOT, long-term oxygen therapy; SGRQ, St George's Respiratory Questionnaire.

\section{MBL deficiency is associated with reduced airway inflammation}

Taking one stable sample from each patient, MBL deficiency was associated with decreased sputum concentrations of EN-RAGE and IL- $1 \beta(p=0.046$ and $p=0.032$, respectively, figure 5); there were no significant differences with other markers of inflammation (serum CRP, serum CD40L, serum P-selectin, sputum Il-8 sputum, sputum TNF- $\alpha$ or sputum myeloperoxidase). MBL deficiency in patients with COPD is associated with less airway inflammation.

\section{MBL binds Haemophilus spp but not $P$. aeruginosa or Streptococcus pneumoniae}

Since MBL deficiency was protective against Haemophilus dysbiosis but not other potential respiratory pathogens, and because MBL binding is required for its effect on phagocytosis, we hypothesised that MBL would bind $H$. influenzae. We investigated the ability of MBL to bind to respiratory pathogens in vitro. We observed that MBL bound to $H$. influenzae, along with Staphylococcus aureus, Burkholderia cenocepacia and Escherichia coli but did not bind to P. aeruginosa, or Streptococcus pneumoniae (figure 6).

\section{DISCUSSION}

This study demonstrates that MBL deficiency is associated with a significantly lower frequency of exacerbations in a large cohort of patients with COPD. In the microbiota subcohort, MBL deficiency was associated with a more diverse microbiota, and in particular, a reduction in Haemophilus spp OTUs. Loss of microbiota diversity with dominance of Haemophilus spp is associated with more severe disease, more frequent exacerbations and greater airway inflammation. Our finding that MBL deficiency is protective against Haemophilus spp infection is consistent with data showing that oxidised MBL disrupts MBL oligomer formation and inhibits phagocytic clearance of Haemophilus spp in COPD alveolar macrophages. ${ }^{12}$ Impaired phagocytosis of Haemophilus spp in COPD macrophages has been previously demonstrated; our data fit with the proposal that MBL has a role in inhibiting clearance of bacteria from the airway. ${ }^{13}{ }^{17} \mathrm{MBL}$ deficiency may be beneficial, by removing an inhibitory factor preventing bacterial phagocytosis. The $23 \%$ reduction in exacerbations we observed in the MBL deficient group is clinically important, and is equivalent to the reduction observed when ICS are added to long-acting $\beta$ agonists in randomised controlled trials. ${ }^{18}$

Our COPD data contrast to CF and bronchiectasis data, where poor outcomes are most strongly associated with $P$. aeruginosa infection. MBL deficiency increases the frequency and severity of P. aeruginosa infections in humans and mice, while studies show lower mortality in patients infected with $\mathrm{H}$. influenzae compared with $P$. aeruginosa and other bacteria. ${ }^{78} 1920$ In the largest bronchiectasis cohort study, colonisation with H. influenzae was associated with a lower frequency of exacerbations compared with P. aeruginosa or Veillonella spp colonisation. ${ }^{21}$ COPD, bronchiectasis and CF have differing microbiota and inflammatory profiles and very different clinical presentations. Therefore, it is plausible MBL deficiency may have different impacts in different diseases. Indeed it has been long speculated that the high MBL deficiency frequency in the population suggests a balanced effect whereby it is harmful under some circumstances but beneficial under others. ${ }^{22}$

The effect in this study appeared to be relatively specific for $H$. influenzae. We found that MBL bound to H. influenzae in vitro, but not to other clinically relevant bacteria except $S$. aureus and B. cenocepacia which are pathogens more frequently isolated in CF than in COPD. The specificity for $H$. influenzae in COPD is therefore likely to reflect the requirement for oxidised MBL to 


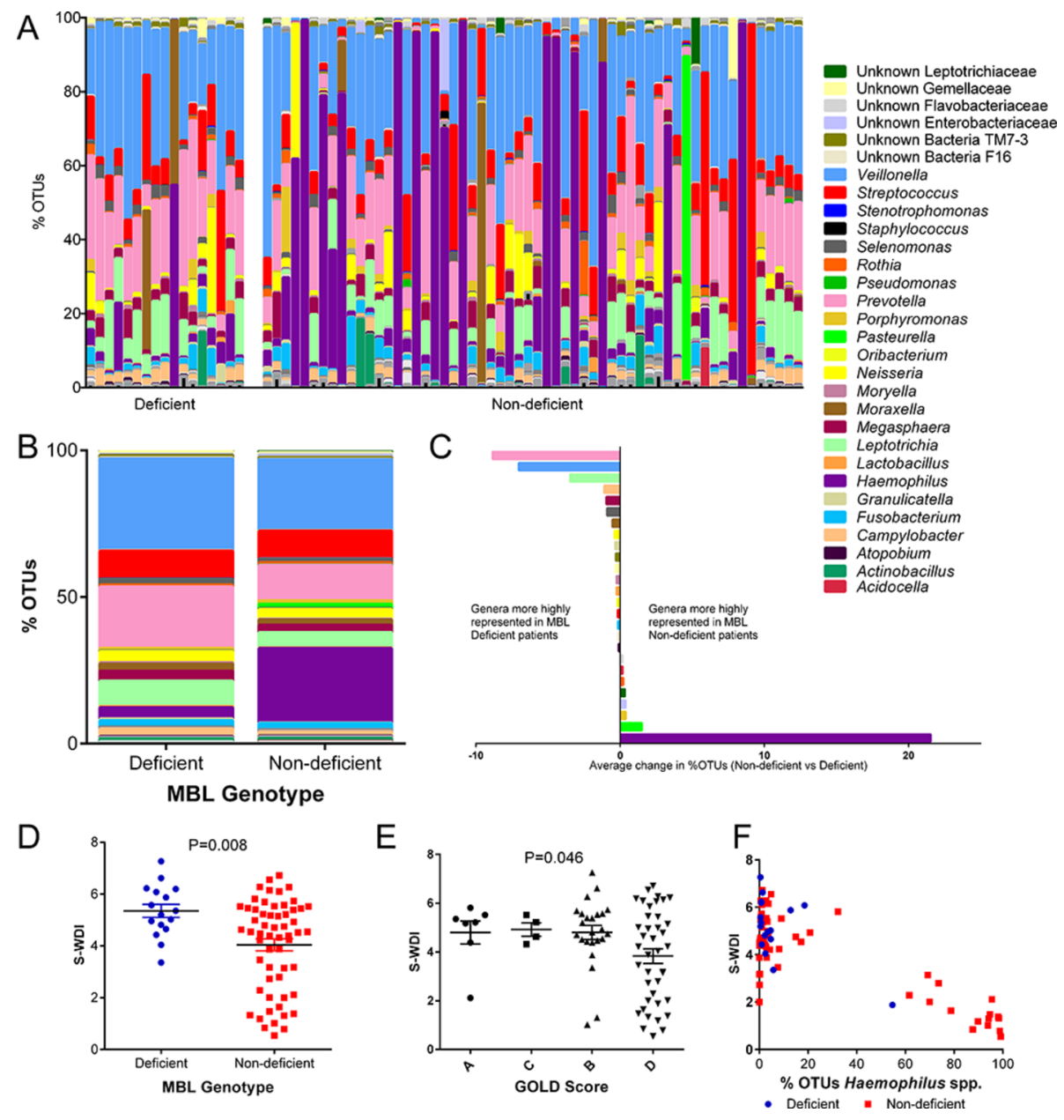

Figure 3 (A) Stacked bar graphs showing the stable microbiota of individual patients, split according to mannose binding lectin (MBL) genotype. Each stacked bar represents one patient when clinically stable; each patient is only represented once, $n=17$ deficient and $n=58$ non-deficient samples. Individual operational taxonomic units (OTUs) representing less than $0.5 \%$ of the total number of OTUs in a sample and not represented in more than 10 samples were excluded from this figure for clarity. (B) Stacked bar graphs showing the average stable microbiota of patients with MBL deficiency and patients without MBL deficiency. Each patient was represented by their first stable sample. (C) Graph showing the average difference in \% OTUs of patients with MBL deficiency and patients without MBL deficiency. For clarity, only genera with an average change of greater than $0.1 \%$ are shown. (D) Reduced microbiota $\alpha$ diversity (Shannon-Wiener Diversity Index (S-WDI)) is associated with a MBL non-deficient genotype by Spearman correlation. Each patient was represented with their first stable sample. (E) Lower S-WDI was associated with a more severe GOLD Score by MannWhitney test. (F) The relationship between \% OTUs identified as Haemophilus spp and S-WDI in stable COPD. Graphs show mean with SE.

bind in order to inhibit phagocytosis, combined with the high prevalence of $H$. influenzae in patients with COPD.

Previous studies on the impact of MBL deficiency in COPD are inconsistent; none have enrolled $>300$ patients without preselection for a subset of patients with COPD, that is, frequent exacerbators, ${ }^{23}$ or had sufficient follow-up time to evaluate exacerbation frequency, whereas we analysed the electronic medical record data from 1796 patients with COPD with an average follow-up of 5.4 years. In the present study, MBL deficiency status was not found to have an effect on decline in $\mathrm{FEV}_{1}$, mortality or hospital admissions for cardiovascular events. The study was not specifically powered for these exploratory end points, however, larger studies or longer follow-up will be needed to thoroughly investigate these outcomes.

Compared with the human gastrointestinal tract microbiota, relatively little is known about the lung microbiota both in health and various lung disease states. The majority of COPD lung microbiota studies have low patient numbers with limited longitudinal sampling, confounded by different sample collection techniques, in populations with varying disease severity or bacterial colonisation status. ${ }^{24-26}$ In comparison, we were able to obtain sputum from a larger cohort of patients with COPD. While there are concerns that the oral microbiota may contaminate sputum, ${ }^{27}$ induced sputum was chosen due to its less invasive nature (compared with bronchoscopy) and the likelihood of obtaining sufficient sample for sequencing. Our results, showing a stable microbiota dominated by Haemophilus, Veillonella, Prevotella and Streptococcus spp, are similar to those obtained by other sample collection methods, ${ }^{24-26}$ and from healthy smokers and non-smokers. ${ }^{27}$ Additionally our data indicating a reduction in $\alpha$ diversity in more severe COPD and with a non-deficient genotype correlate with other studies which have compared the lung microbiota of different COPD stages with healthy smokers and non-smokers. ${ }^{2}$ We observed relatively few Pseudomonas genera OTUs. ${ }^{24-26}$ Our study was community based, examining a patient population with less severe COPD than other studies, and may be more representative of the wider patient population with COPD since Pseudomonas spp are known colonisers in more severe COPD and 
A

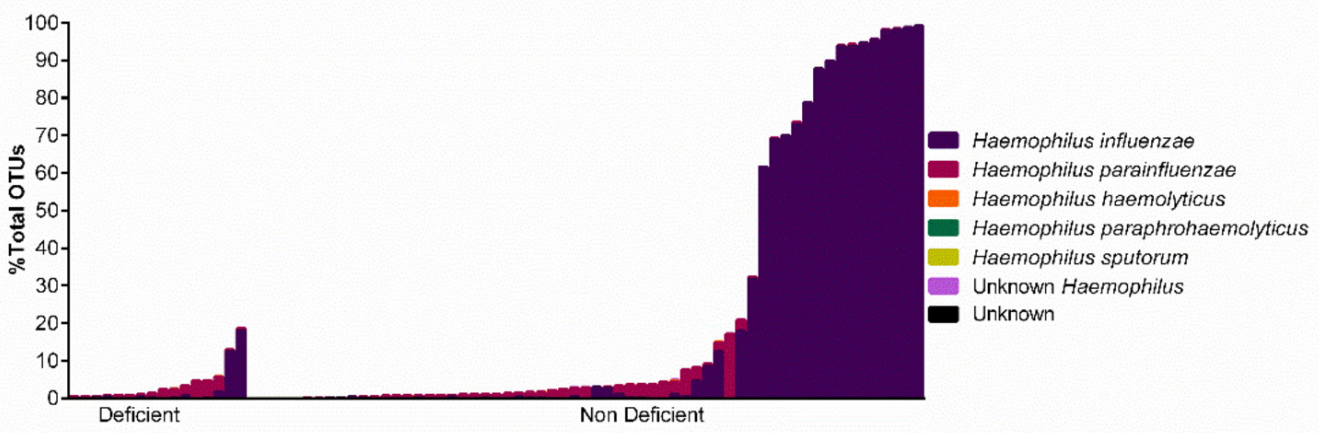

B

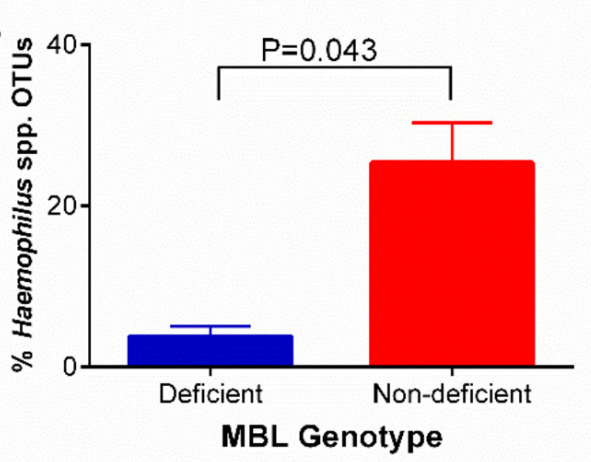

D

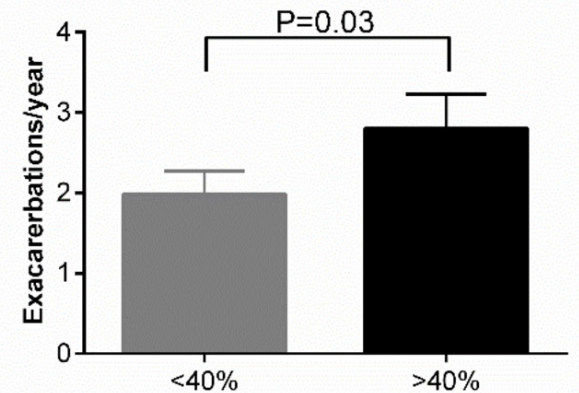

Haemophilus spp. OTUs

F

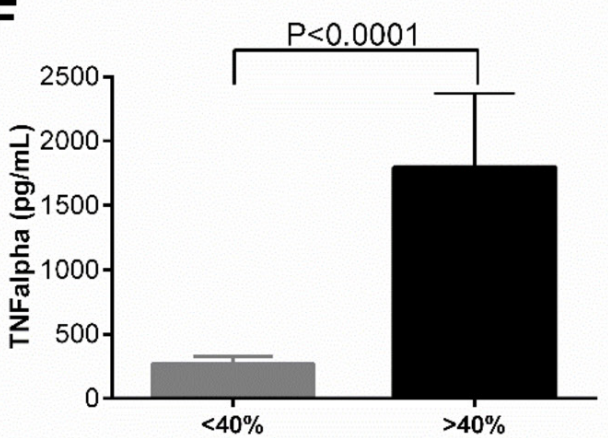

Haemophilus spp. OTUs
C

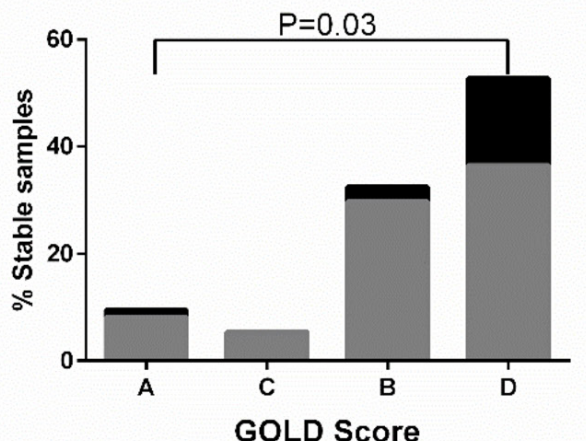

$>40 \%$ Haemophilus $<40 \%$ Haemophilus

E

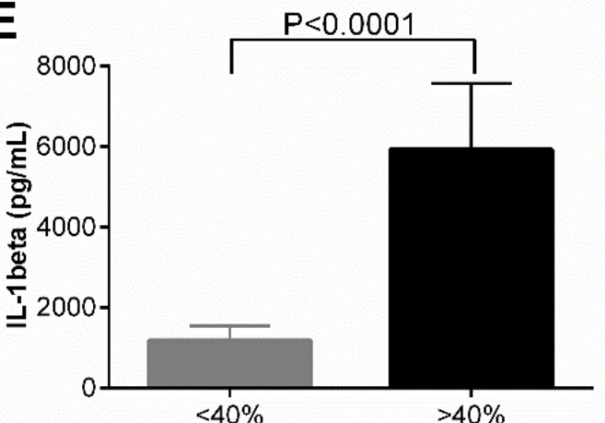

Haemophilus spp. OTUs

G

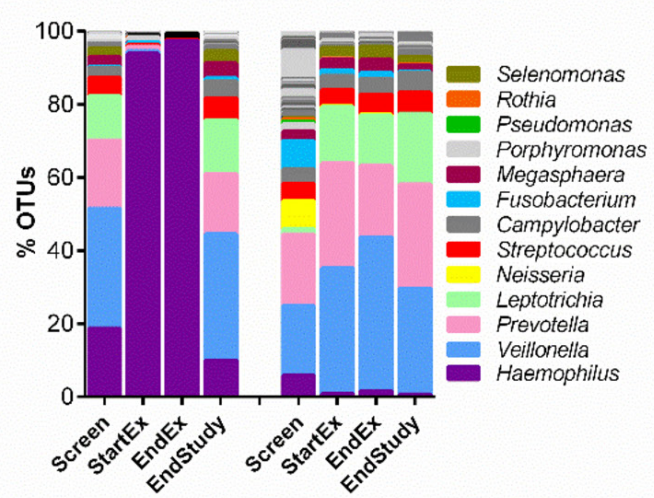

Figure 4 (A) Graph showing only the Haemophilus spp operational taxonomic units (OTUs) from each sample further identified to species level indicating the dominant Haemophilus spp was Haemophilus influenzae; all samples were from stable patients and each patient was only represented once. (B) Mean percentage of OTUs identified as Haemophilus spp in patients with mannose binding lectin (MBL) deficiency and patients without MBL deficiency. (C) One stable sample per patient classified according to GOLD 2011 Score, colour coded according to whether the sample had $>40 \%$ Haemophilus spp OTUs. (D) The percentage of OTUs identified as Haemophilus spp compared with the number of exacerbations per year. (E,F) Sputum IL-1 $\beta$ and TNF $\alpha$ concentrations from stable patients with COPD (one sample per patient) stratified according to percentage of Haemophilus spp OTUs in sample. (G) Example of an exacerbation dominated by Haemophilus spp compared with a non-Haemophilus spp dominated exacerbation. Statistical analysis of microbiota data was carried out using the non-parametrical Spearman correlation and Mann-Whitney test, where appropriate; graphs show mean with SE. 


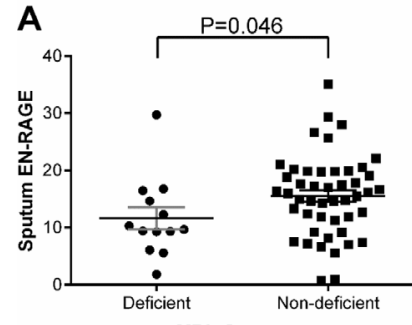

MBL Genotype

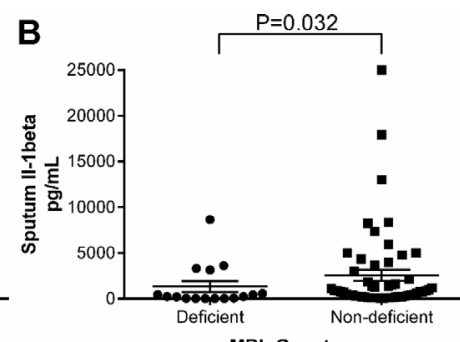

MBL Genotype
Figure 5 (A) Sputum EN-RAGE (S100A12) levels and (B:) IL-1 $\beta$ levels from stable patients with COPD grouped according to mannose binding lectin (MBL) genotype. Statistical analysis was carried out using the non-parametrical Mann-Whitney test.

bronchiectasis. ${ }^{28} 29$ We observed a clear correlation between clinical disease severity and microbiota diversity using S-WDI, similar to the reduced lung microbiota diversity shown to be associated with more severe COPD in other studies. ${ }^{25}$

This study is the first to have sufficient statistical power to demonstrate correlations between microbiota diversity and disease severity or inflammatory profiles, both during clinical stability and at exacerbation. In addition, this is the first study to show a significant genetic modifier of the lung microbiota, although whether this modification is direct (through modulation of host immunity) or indirect (through protecting against antibiotic use for example), is unclear. This finding needs to be validated, but it is logical to expect that host genetics can influence the composition of the microbiota.

Proinflammatory IL-1 $\beta$, one of the most useful discriminators of bacterial infection in COPD, ${ }^{16}$ promotes neutrophil recruitment. Neutrophilic inflammation contributes to COPD progression, and is poorly responsive to ICS. ${ }^{30}$ We identified higher levels of IL-1 $\beta$ and the RAGE ligand S100A12 (EN-RAGE) in sputum from patients without MBL deficiency. Although elevated IL-1 $\beta$ may be a reflection of the lower bacterial diversity in individuals without MBL deficiency, it has previously been shown that exposure to $\mathrm{MBL}$ can promote the increased release of IL-1 $\beta$ in response to Gram-negative pathogens like Neisseria meningitides or by lipopolysaccharide, suggesting that MBL can be directly responsible for increased inflammation, independent of its effect as an opsonin. ${ }^{31}$

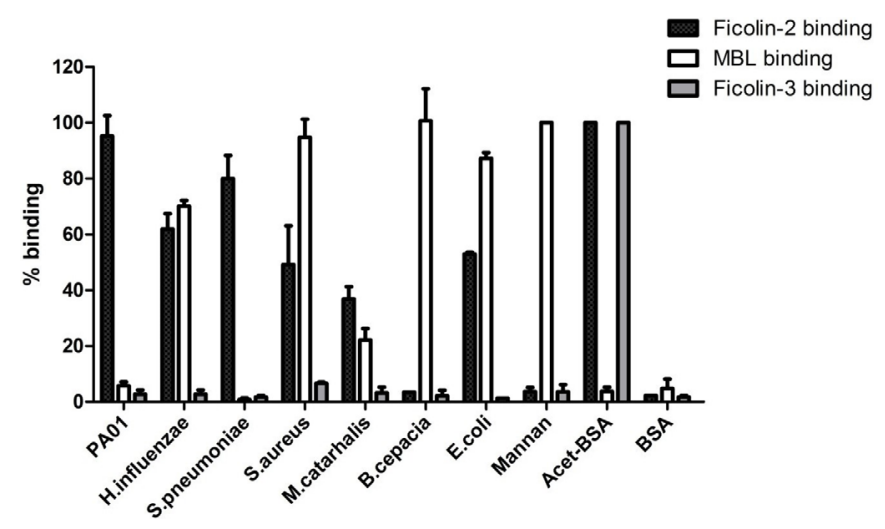

Figure 6 Binding of lectin pathway components to respiratory pathogens. Binding is expressed as a percentage of the positive control (acetylated bovine serum albumin for ficolin-2/ficolin-3 and mannan for mannose binding lectin (MBL)). Binding assays represent the mean with $\mathrm{SE}$ of three independent experiments performed in duplicate.
Limitations of the Go-TARDIS study include the use of primary care diagnosis, hospitalisation records and prescription data to identify exacerbations, however, these are widely used epidemiological methods. The patient population consisted of primarily early COPD with preserved lung function, and hence the generalisability of these data to later stage COPD would need to be confirmed in other studies. The sample size in the microbiota subcohort study was relatively small, reflecting the fact that MBL deficiency affects only $10 \%$ of the population; larger, multinational studies would be valuable. Nevertheless, this represents the largest study of the microbiota in COPD to date and the largest study of the impact of genetic MBL deficiency in COPD.

In conclusion, we have shown, using a large primary care based genetic study and a nested microbiota subcohort study, MBL deficiency in COPD is protective against the risk of exacerbations and is associated with a more diverse lung microbiota. MBL genotype is a modifier of disease phenotype and exacerbation risk in COPD.

\section{Author affiliations}

${ }^{1}$ Scottish Centre for Respiratory Research, School of Medicine, University of Dundee, Ninewells Hospital and Medical School, Dundee, UK

${ }^{2}$ Tayside Respiratory Research Group, Clinical Research Centre, Dundee, UK ${ }^{3}$ Dundee Epidemiology and Biostatistics Unit, University of Dundee, Ninewells Hospital and Medical School, Dundee, UK

${ }^{4}$ Pat MacPherson Centre for Pharmacogenetics and Pharmacogenomics, University of Dundee, Ninewells Hospital and Medical School, Dundee, UK

${ }^{5} \mathrm{~S}$ chool of Medicine, Centre for Experimental Medicine, Dentistry and Biomedical

Sciences, Queen's University, Belfast, UK

${ }^{6}$ Department of Immunology, NHS Tayside, Ninewells Hospital Department of Medicine, Dundee, Dundee, UK

${ }^{7}$ National Heart and Lung Institute, Imperial College London, London, UK

Acknowledgements The authors thank the Health Informatics Centre, University of Dundee for managing and supplying anonymized data and NHS Tayside, the original data source. The authors also thank Mike Lonergan and Peter T Donnan for additional statistical support.

Contributors All authors participated in study design, data analysis and interpretation of the data. All authors were involved in writing and revising the article prior to submission.

Funding Chief Scientist Office, Scotland (ETM/262). JDC is supported by the GSK/ British Lung Foundation Chair of Respiratory Research and a fellowship from the Wellcome Trust (099084/Z/12/Z) . GoSHARE was supported by the Wellcome Trust (099177/Z/12/Z).

Competing interests SEM is now an employee of the Wellcome Trust. All other authors have no competing interest to decline.

Patient consent Obtained.

Ethics approval East of Scotland Research Ethics Committee.

Provenance and peer review Not commissioned; externally peer reviewed.

Data sharing statement Data are available and can be accessed through the Health Informatics Centre at the University of Dundee.

Open Access This is an Open Access article distributed in accordance with the Creative Commons Attribution Non Commercial (CC BY-NC 4.0) license, which permits others to distribute, remix, adapt, build upon this work non-commercially, and license their derivative works on different terms, provided the original work is properly cited and the use is non-commercial. See: http://creativecommons.org/ licenses/by-nc/4.0/

(c) Article author(s) (or their employer(s) unless otherwise stated in the text of the article) 2018. All rights reserved. No commercial use is permitted unless otherwise expressly granted.

\section{REFERENCES}

1 Vestbo J, Hurd SS, Agustí AG, et al. Global strategy for the diagnosis, management, and prevention of chronic obstructive pulmonary disease: GOLD executive summary. Am J Respir Crit Care Med 2013;187:347-65.

2 Erb-Downward JR, Thompson DL, Han MK, et al. Analysis of the lung microbiome in the "healthy" smoker and in COPD. PLoS One 2011;6:e16384. 
3 Vestbo J, Agusti A, Wouters EF, et al. Evaluation of COPD Longitudinally to Identify Predictive Surrogate Endpoints Study Investigators. Should we view chronic obstructive pulmonary disease differently after ECLIPSE? A clinical perspective from the study team. Am J Respir Crit Care Med 2014;189:1022-30.

4 Casanova C, de Torres JP, Aguirre-Jaíme A, et al. The progression of chronic obstructive pulmonary disease is heterogeneous: the experience of the BODE cohort. Am J Respir Crit Care Med 2011;184:1015-21.

5 Fidler KJ, Hilliard TN, Bush A, et al. Mannose-binding lectin is present in the infected airway: a possible pulmonary defence mechanism. Thorax 2009;64:150-5.

6 Garred P, Honoré C, Ma YJ, et al. MBL2, FCN1, FCN2 and FCN3-The genes behind the initiation of the lectin pathway of complement. Mol Immunol 2009;46:2737-44.

7 Chalmers JD, McHugh BJ, Doherty C, et al. Mannose-binding lectin deficiency and disease severity in non-cystic fibrosis bronchiectasis: a prospective study. Lancet Respir Med 2013;1:224-32.

8 Garred P, Pressler T, Madsen HO, et al. Association of mannose-binding lectin gene heterogeneity with severity of lung disease and survival in cystic fibrosis. J Clin Invest 1999;104:431-7.

9 Yang IA, Seeney SL, Wolter JM, et al. Mannose-binding lectin gene polymorphism predicts hospital admissions for COPD infections. Genes Immun 2003;4:269-74.

10 Mandal J, Malla B, Steffensen R, et al. Mannose-binding lectin protein and its association to clinical outcomes in COPD: a longitudinal study. Respir Res 2015;16:150.

11 Eagan TM, Aukrust P, Bakke PS, et al. Systemic mannose-binding lectin is not associated with chronic obstructive pulmonary disease. Respir Med 2010;104:283-90

12 Tran $\mathrm{HB}$, Ahern J, Hodge G, et al. Oxidative stress decreases functional airway mannose binding lectin in COPD. PLoS One 2014;9:e98571.

13 Donnelly LE, Barnes PJ. Defective phagocytosis in airways disease. Chest 2012;141:1055-62.

14 Seemungal TA, Donaldson GC, Paul EA, et al. Effect of exacerbation on quality of life in patients with chronic obstructive pulmonary disease. Am J Respir Crit Care Med 1998;157:1418-22.

15 Altman DG, Bland JM. Interaction revisited: the difference between two estimates. BMJ 2003;326:219.

16 Bafadhel M, McKenna S, Terry S, et al. Acute exacerbations of chronic obstructive pulmonary disease: identification of biologic clusters and their biomarkers. Am J Respir Crit Care Med 2011;184:662-71.

17 Berenson CS, Garlipp MA, Grove L, et al. Impaired phagocytosis of nontypeable Haemophilus influenzae by human alveolar macrophages in chronic obstructive pulmonary disease. J Infect Dis 2006;194:1375-84.
18 Dransfield MT, Bourbeau J, Jones PW, et al. Once-daily inhaled fluticasone furoate and vilanterol versus vilanterol only for prevention of exacerbations of COPD: two replicate double-blind, parallel-group, randomised controlled trials. Lancet Respir Med 2013;1:210-23.

19 Møller-Kristensen M, Ip WK, Shi L, et al. Deficiency of mannose-binding lectin greatly increases susceptibility to postburn infection with Pseudomonas aeruginosa. J Immunol 2006;176:1769-75.

20 Chalmers JD, Goeminne P, Aliberti S, et al. The bronchiectasis severity index. An international derivation and validation study. Am J Respir Crit Care Med 2014; 189:576-85.

21 Rogers GB, Zain NM, Bruce KD, et al. A novel microbiota stratification system predicts future exacerbations in bronchiectasis. Ann Am Thorac Soc 2014:11:496-503.

22 Eisen $\mathrm{DP}$, Osthoff $\mathrm{M}$. If there is an evolutionary selection pressure for the high frequency of MBL2 polymorphisms, what is it? Clin Exp Immunol 2014;176:165-71.

23 Albert RK, Connett J, Curtis JL, et al. Mannose-binding lectin deficiency and acute exacerbations of chronic obstructive pulmonary disease. Int I Chron Obstruct Pulmon Dis 2012;7:767-77.

24 Aguirre E, Galiana A, Mira A, et al. Analysis of microbiota in stable patients with chronic obstructive pulmonary disease. APMIS 2015;123:427-32.

25 Garcia-Nuñez M, Millares L, Pomares X, et al. Severity-related changes of bronchial microbiome in chronic obstructive pulmonary disease. J Clin Microbiol 2014;52:4217-23.

26 Millares L, Ferrari R, Gallego M, et al. Bronchial microbiome of severe COPD patients colonised by Pseudomonas aeruginosa. Eur J Clin Microbiol Infect Dis 2014:33:1101-11.

27 Morris A, Beck JM, Schloss PD, et al. Comparison of the respiratory microbiome in healthy nonsmokers and smokers. Am J Respir Crit Care Med 2013;187:1067-75.

28 Boixeda R, Almagro P, Díez-Manglano J, et al. Bacterial flora in the sputum and comorbidity in patients with acute exacerbations of COPD. Int I Chron Obstruct Pulmon Dis 2015;10:2581-91.

29 Aydemir Y, Aydemir Ö, Kalem F. Relationship between the GOLD combined COPD assessment staging system and bacterial isolation. Int I Chron Obstruct Pulmon Dis 2014;9:1045-51.

30 Brusselle G, Bracke K. Targeting immune pathways for therapy in asthma and chronic obstructive pulmonary disease. Ann Am Thorac Soc 2014;11(Suppl 5):S322-S328.

31 sprong T, Jack DL, Klein NJ, et al. Mannose binding lectin enhances IL-1beta and IL-10 induction by non-lipopolysaccharide (LPS) components of Neisseria meningitidis. Cytokine 2004;28:59-66. 